\title{
Color Image Segmentation using Type-2 Fuzzy Sets
}

\author{
Saikat Maity, Jaya Sil, Member, IEEE
}

\begin{abstract}
Domain knowledge of real life problems are often uncertain, imprecise and inexact, therefore create difficulty in decision making while solving by conventional approaches. Among various methods of handling uncertainties, fuzzy logic has been most intensively studied almost over four decades. Fuzzy logic (FL) explores human reasoning power using linguistic terms, which are modeled as type-1 fuzzy sets and represented by membership functions (MF). However, the MF of type-1 fuzzy set is crisp and cannot tackle all kind of uncertainties. Introducing type-2 fuzzy sets, an extension of type-1 simplifies the problem where the MF is itself fuzzy with three dimension representations. Moreover, a type-2 fuzzy set maps elements of a crisp domain to type-1 fuzzy numbers bounded in the range $[0,1]$. Research on type-2 fuzzy sets is still in its infancy and very recently has been applied on emerging areas that need development of more efficient and robust systems. The aim of the review paper is to describe type-2 fuzzy systems for managing uncertainties, presenting the frontier research areas where type-2 fuzzy logic has been applied and proposes algorithm on application of type-2 fuzzy sets in color image segmentation.
\end{abstract}

Index Terms - Fuzzy Logic, Membership Function, Foot-Print of Uncertainty, Interval Type-2 fuzzy sets.

\section{INTRODUCTION}

Information systems are often poorly defined, which create difficulty in representing concepts and extracting important features used to solve the problems. Type 1 (T1) fuzzy sets (FS) are pioneered by Zadeh [1] has been around for more than four decades, and yet not able to handle all kinds of uncertainties appearing in real life problem domain. The above statement sounds paradoxical because the word fuzzy has the connotation of uncertainty. The extension of T1 fuzzy systems, in particular type-2 (T2) accommodate the system uncertainties and minimize its effect considerably in decision making. However, T2 FS is difficult to understand and explain. The relationship between $\mathrm{T} 1$ and $\mathrm{T} 2$ can be stated, "if all uncertainties disappear, $\mathrm{T} 2$ reduces to $\mathrm{T} 1$, in much the same way when randomness disappear probability reduces to determinism".

Although T1 fuzzy logic has been used in many applications, its application to rule-based systems most

Manuscript received May 5, 2009.

Saikat Miaty is head of the department of the Dr.B.C.Roy Engineering College, in the department of Information Technology, Durgapur - 6 India, phone: +919932252306; fax: +913432503121

Dr.Jaya Sil is with Bengal Engineering Science \& University, Howrah, India.She is now Head of the department in the Department of Computer Science and Engineering in B.E.S.U and also the member of IEEE.. significantly demonstrates its importance as a powerful design methodology to tackle uncertainties. A fuzzy logic system (FLS), described completely in terms of T1 fuzzy sets is called T1 fuzzy logic system (T1FLS), whereas a FLS with at least one T2 fuzzy set is called T2 fuzzy logic system (T2FLS). T1FLSs cannot directly handle rule uncertainties because T1 fuzzy sets are certain. T2FLSs, on the other hand, are very useful in circumstances where it is difficult to determine an exact membership function of a fuzzy set. Such cases are handled by rule uncertainties and measurement uncertainties. Like T1FLS, T2 has wide applications and truly speaking the potential of $\mathrm{T} 2$ systems outperforms $\mathrm{T} 1$ in most of the cases. The aim of the review paper is to describe T2 fuzzy systems for managing uncertainties, identifying the frontier research areas where T2 fuzzy logic are applied and proposes algorithm on application of type-2 fuzzy sets in color image segmentation.

\section{MODELING UNCERTAINTY USING FUZZY LOGIC}

comes in many guises and is independent of the kind of fuzzy logic (FL) or any kind of methodology one uses to handle it [2]. Uncertainty involved in any real life problem-solving situation, due to information deficiency of various forms. One of the best sources for general discussions about uncertainty is found in [3]. Two types of uncertainties, randomness and fuzziness exist, where probability theory is associated with the former and FS with the latter. Fuzziness (or vagueness) generally recognizes uncertainty resulting from the imprecise boundaries of fuzzy sets, nonspecificity (or imprecision) connected with sizes (cardinalities) of relevant sets of alternatives and strife (or discord), which expresses conflicts among the various sets of alternatives. The T1FLSs cannot directly handle rule uncertainties because they use T1 fuzzy sets that are certain. A FLS needs some measure to capture uncertainties than just a single number. The extended FL, named as T2FL able to handle uncertainties by modeling and subsequently minimizing their effects. T2FLSs move the world of FLS into a fundamentally new and important direction. T2 fuzzy logic provides the measure of dispersion and fundamental to the design of systems that include linguistic or numerical uncertainties translating into rules. T2 fuzzy set is a natural framework for handling both randomness and fuzziness. It is the third dimension of T2 membership function (MF) that allows us to evaluate the model uncertainties based on the knowledge of the problem at hand.

Linguistic and random uncertainties flow through a T2FLS and their effects can be evaluated using the 
defuzzified and the type-reduced output of that system. The type-reduced output can be interpreted as providing a measure of dispersion about the defuzzified output. A T2FLS has more design degrees of freedom than does a T1FLS because respective T2 fuzzy sets are described by more parameters compare to T1 fuzzy sets and so a T2FLS has the potential to outperform a T1FLS, though, to date, no mathematical proof can substantiate the claim. However, in every application of T2FLS, it has been observed that T2FLS performs better compare to T1FLS.

\section{EXTENSION OF TYPE1 FUZZY LOGIC SYSTEM LOGIC}

For ready reference to the readers, T1 fuzzy set is described very briefly.

The knowledge used to construct the rules of a FLS is uncertain and the following way uncertainty can occur in a system:

34) The words used in antecedents and consequents of the rules can mean different things to different people.

35) Consequents obtained by polling a group of experts often are different for the same rule because the experts are not necessarily be in agreement and opinions may change with time.

36) Noisy training data.

Once the rules are established, fuzzy expert system (FES) can be viewed as a (non-linear) mapping from inputs to outputs using fuzzy If-Then rules provided by experts or can be extracted from numerical data. A typical rule: If arterial $\mathrm{pH}$ is low and venous $\mathrm{pH}$ is low, Then academia is severe. In an FES, the linguistic terms are mapped as antecedent and consequents of the rules. In general, many different functions may be used to generate T1 fuzzy subsets among which MF is a common choice. Sigmoidal and products of sigmoidal MFs are the natural choice given in equation (1) and described in fig 1.

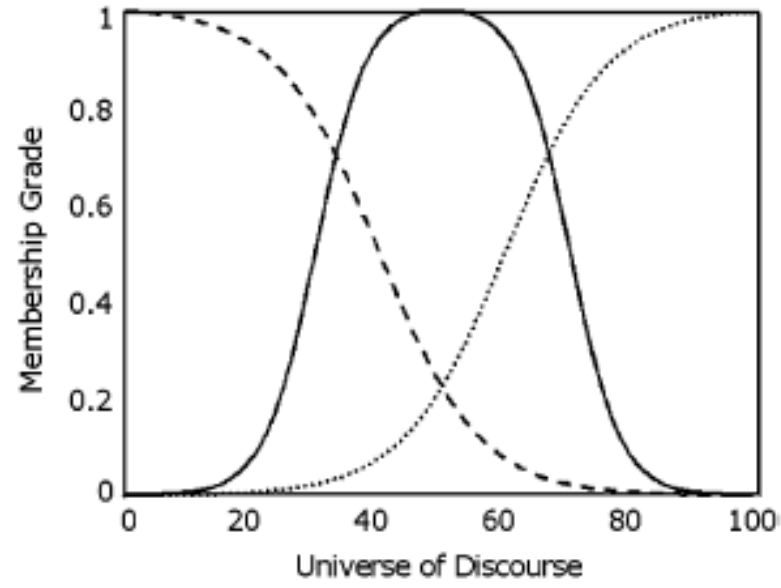

Fig. 1 Three Illustrative T1 Sigmoidal membership function $\mu(x 1)=1 / 1+e^{(x+c) / w}$

(1a)

In equation (1a), $c$ the centre of sigmoid, $x 1$ the variable and $w$ describe the width of the sigmoid function.

The other sigmoidal function is

$\mu(x 2)=1 / 1+e^{(x-c 2) / w}$

(1b)

Middle MF of fuzzy set may be generated by the product of two such sigmoid, given in (1c).

$\mu(x)=\left(1 / 1+e^{(c 1-x) / w}\right)\left(1 / 1+e^{(x-c 2) / w}\right)$ where $\mathrm{c} 1$ is the centre of the increasing sigmoid, $\mathrm{c} 2$ the centre of the decreasing sigmoid, and the steepness of the slopes, or "width," of the sigmoid which in general be different for the increasing and decreasing sigmoid. Symmetrical middle sigmoids, computationally efficient can be expressed using (1d).

$\mu(x)=\left(1 / 1+e^{(c-5 w-x) / w}\right)\left(1 / 1+e^{(x-c-5 w) / w}\right)$

The concept of T2 fuzzy set was introduced by Zadeh as an extension of T1 fuzzy set whose grade of membership value itself fuzzy. A T2 membership grade[4] can be any subset in the primary membership and corresponding to each primary membership, there is a secondary membership that defines the possibilities for the primary membership. T2 fuzzy sets allow us to handle linguistic uncertainties, since "words can mean different things to different people."

A fuzzy relation of higher type (e.g., T2) has been regarded as one way to increase the fuzziness of a relation and according to Hisdal [5] "increased fuzziness in a description means increased ability to handle inexact information in a logically correct manner." Mizumoto and Tanaka [6] studied the set theoretic operations of T2 sets, properties of membership grades of such sets, and examined the operations of algebraic product and algebraic sum. Dubois and Prade [7] discussed fuzzy valued logic and obtain a formula for the composition of $\mathrm{T} 2$ relations as an extension of the T1 sup-star composition [8]. However, this formula is only for minimum norm [9]. A general formula for the extended sup-star composition of $\mathrm{T} 2$ relation is presented in [10]. Based on this formula, Karnik and Mendel established a complete T2 fuzzy logic theory to handle uncertainties in FLS parameters. Similar to a T1FLS, a T2FLS includes fuzzifier, rule base, fuzzy inference engine, and output processor, described in figure 2 . The output processor includes type-reducer and defuzzifier, which generates a T1 fuzzy set output. A T2FLS is characterized by If-Then rules, but its antecedent or consequent sets are now T2 fuzzy set. Generally, T2FLS is computationally intensive due to type-reduction operation which simplified considerably when secondary membership functions become treated as interval sets having membership values either zero or one and called interval T2 sets. The T2 fuzzy logic consisting of secondary MF as interval sets, termed as "interval type-2 FLS." Use of interval sets in fuzzy logic has been discussed in Hisdal, Schwartz, and Turksen approach [11]. The most commonly used fuzzifier is a singleton but such a fuzzifier is not adequate when data get corrupted by measurement noise. In such cases, a nonsingleton fuzzifier that treats each measurement as a fuzzy number should be used for fuzzification.

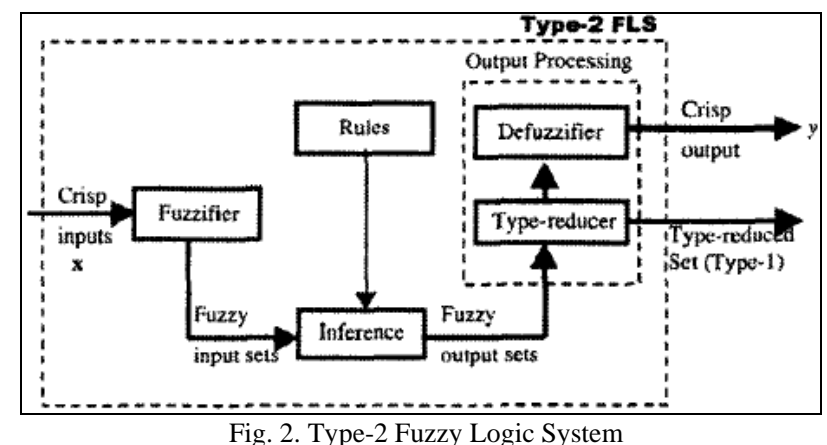

Fig. 2. Type-2 Fuzzy Logic System 
The theory and applications of T1FLS with nonsingleton fuzzifier are presented in Turksen approach [12] where the input is fuzzified into a T1 fuzzy set (e.g., Gaussian). To interpret the Gaussian MF the statistical knowledge (mean and variance) of the noise is supplied or estimated but in many cases, these values are not known ahead of time and may not be estimated from the data. Instead, we only have some linguistic information about the noise, like very noisy, moderately noisy, or approximately no noise. Fuzzification of such input as T1 fuzzy set does not serve the purpose because T1 MFs cannot fully represent the uncertainty associated with the linguistic information. In order to handle such uncertainties the input should be fuzzified into a T2 fuzzy set to frame in a nonsingleton T2FLS. The T1 determines input/output (I/O) space partition, antecedent and consequent variables, the number of If-Then rules (which are determined by the I/O space partition) and the number and initial locations of membership functions. The latter identifies a feasible set of parameters under the given structure. Tuning of parameters of a T1FLS is possible because its output can be expressed as a closed-form mathematical formula. Optimization methods for performing this have been extensively studied. Unfortunately, the output of a T2FLS cannot be represented by a closed-form mathematical formula and hence, there is an additional level of complexity associated with tuning its parameters. Simplifying T2 fuzzy set to interval T2 fuzzy set, the computational complexity can be significantly reduced in the appropriate applications. The reduction on the computational complexity is due to the property that all the secondary grades for an interval T2 fuzzy set are uniformly weighted (i.e., all equal to one). In general, the management of uncertainty using T2 fuzzy sets may be applied to various fields where we cannot obtain satisfactory performance with ordinary T1 fuzzy sets.

\section{SCOPE OF WORK}

Image segmentation is one of the most difficult low-level image analysis tasks mainly due to vagueness in representation of images. In earlier works[13], image segmentation was applied in monochrome color images. However, the color uncertainty was not inherited within the gray level images and later applied on red, green, blue (RGB) color space. Two main image segmentation techniques are described in the literature; region reconstruction where image plane is being analyzed using region growing process [14] and color space analysis where the color of each pixel is represented in the designated color space. Many authors have tried to determine the best color space for some specific color image segmentation problems [15], however, there does not exist a unique color space for all segmentation problems. Computational complexity may increase significantly with reference to $\mathrm{C}(\mathrm{Cyan})$, M(Magenta),Y(Yellow), K(contrast) (CMYK) [16] color space in comparison with gray scale image segmentation. Classically, the RGB color space has been chosen for color image segmentation where a point in the image is defined by the color component levels of the corresponding R, G and B pixels. However, while the region growing techniques tend to over-segment the images, the color space analysis methods are not robust enough to significance appearance changes because they do not include any spatial information. Fuzzy logic is considered to be an appropriate tool for image analysis, applicable in CMYK and particularly for gray scale segmentation [18]. Recently, fuzzy region oriented techniques for color image segmentation based on fuzzy logic with T1 fuzzy sets are presented in [19]. Other techniques [17] have been presented to perform color clustering in a color space [20], based on T1 fuzzy sets and fuzzy homogeneity (or fuzzy entropy). The major concern of these techniques is spatial ambiguity among pixels, which has inherent vagueness rather than randomness. However, there still remain some sources of uncertainties with the meanings of the words used for noisy measurements and the data used to tune the parameters of T1 fuzzy sets may be noisy too. The new concept of evidence theory allows to tackling imprecision in model uncertainty used in pattern classification, and produce good results in segmentation [22], although this technique based on CMYK model is not often used. The amount of uncertainty is evaluated using the approach proposed by Klir [21] where he generalizes the Shannon entropy to belief functions using two uncertainty measures, mainly the non-specificity measure and the discord measure. The robust method using T2 fuzzy set is another approach for handling uncertainty in image analysis has not been applied yet through it can take into account three kinds of uncertainty, namely fuzziness, discord and nonspecificity [23]. T2 fuzzy sets have grades of membership value that are themselves fuzzy. Hence, the membership function of a T2 fuzzy set is three dimensional, and it is the new third dimension that provides new design degrees of freedom for handling uncertainty. In the proposed work, color uniformity has been considered a relevant criterion to partition an image into significant regions using fuzzy entropy approach to take into account simultaneously the color and spatial properties of the pixels. For high resolution based pictures new scheme has been proposed in the paper based on CMYK color model using T2 fuzzy sets that tackles total uncertainty inherent in the images.

\section{PRELIMIRERS OF TYPE-2 FUZZY SYSTEM}

According to Gertrude Stein, "A rule is a rule is a rule....". The rule doesn't change, what does change is the way in which we model a rule's antecedent and consequent as fuzzy sets. The term "fuzzy set" is general that include T1 and T2 fuzzy sets (and even higher-type fuzzy sets). All fuzzy sets are characterized by MFs. A T1 fuzzy set is characterized by a two-dimensional MF, whereas a T2 fuzzy set is characterized by a three-dimensional MF. Let us take an example of linguistic variable "speed". Different values of the variable like "very high speed", "high speed", "low speed" signify the crisp value. One approach to using the 100 sets of two endpoints is to average the endpoint data and use the average values for the interval associated with "Speed". A triangular (or other shape) MF has been constructed whose base endpoints (on the $x$-axis) are at the two average values and whose apex is midway between the two endpoints. The T1 triangular MF has been represented in two dimensions and expressed mathematically as

$\{(x, \operatorname{MF}(x)) \mid x$ an element of $X\}$ 
However, the MF completely ignores the uncertainties associated with the two endpoints. A second approach is to make use of the average values and the standard deviations for the two endpoints. The approach blurs the location in between the two endpoints along the $x$-axis. Now the triangles are located in such a way so that their base endpoints can be anywhere in the intervals along the $x$-axis associated with the blurred average endpoints, which leads to a continuum of triangular MFs on the $x$-axis. Thus whole bunch of triangles, all having the same apex point but different base points are obtained as shown in figure 4 . Suppose there are exactly $N$ such triangles, and at each value of $x, \mathrm{MF}$ 's are represented by: $\mathrm{MF}_{1}(x), \mathrm{MF}_{2}(x), \quad \ldots, \mathrm{MF}_{N}(x)$ Weight is assigned to each of the membership values, say $w_{x 1}, w_{x 2}, \ldots, w_{x N}$, representing the possibilities associated with each triangle at a particular value of $x$.

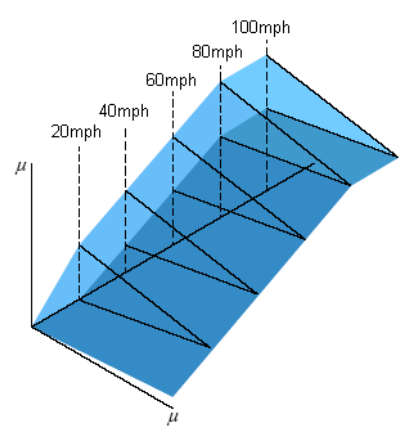

Fig. 3 3-D Representation of T2 FS "Speed"

The resulting T2 MF is expressed using (3)

$\left\{\left(x,\left\{\left(M F_{i}(x), w_{x i}\right) \mid i=1, \ldots, N\right\} \mid x\right.\right.$ an element of $\left.X\right\}$

(3)

Another way to represent the membership value: $\{(x, \operatorname{MF}(x$, $w) \mid x$ an element of $X$ and $w$ an element of $\left.J_{x}\right\}$ where $\operatorname{MF}(x$, $w)$ is a $\mathrm{T} 2 \mathrm{MF}$ which is three-dimensional.

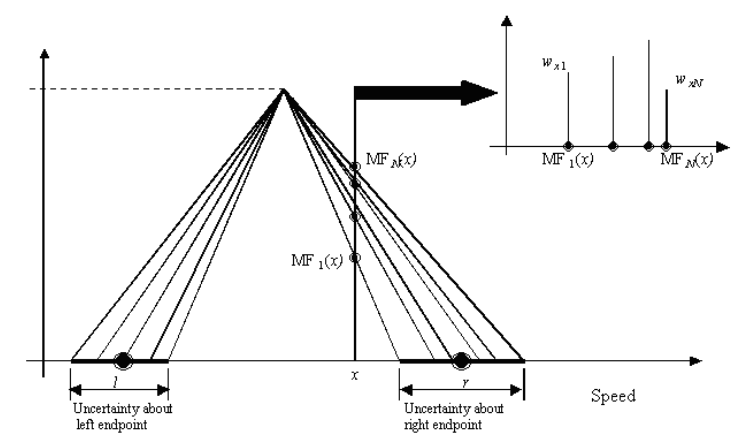

Fig. 4 Triangular MFs (base endpoints $l$ and $r$ ) with Uncertain Intervals Another way to visualize T2 fuzzy sets is to plot their footprint of uncertainty (FOU).

\section{A. Footprint of Uncertainty}

In $\mathrm{T} 2, \operatorname{MF}(x, w)$ can be represented in a two-dimensional $x-w$ plane, consisting of only the permissible (sometimes called "admissible") values of $x$ and $w$. It implies that $x$ is defined over a range of values (its domain), say, $X$ while $w$ is defined over its range of values (its domain), say, $W$. An example of an FOU is shown in figure 5, representing FOU for a Gaussian MF. The standard deviation of the MF is known with perfect certainty but mean, $m$, is uncertain and varies anywhere in the interval from $m_{1}$ to $m_{2}$, which can be expressed as $m\left[m_{1}, m_{2}\right]$. Uncertainty in the primary memberships of a T2 fuzzy set, $\tilde{A}$, consists of a bounded region and called the footprint of uncertainty (FOU). FOU is the union of all primary memberships $\left(J_{x}\right)$, given in (4).

$\operatorname{FOU}(\tilde{A})=\bigcup_{x \in X} J x$

The term FOU is very useful, since it focuses our attention on the uncertainties inherent in a specific $\mathrm{T} 2$ membership function, whose shape is a direct consequence of the nature of the uncertainty, described in figure 6 . Moreover, FOU provides a very convenient verbal description of the entire domain of support for all the secondary grades of a T2 membership function. It depicts a T2 fuzzy set graphically in two-dimensions instead of three dimensions, and therefore overcome the difficulty. T1 and T2 MFs are distinguished using the new terminology FOU. The shaded FOUs indicate that there is a distribution that sits on top of it - the new third dimension of T2 fuzzy sets. Shape of the distribution depends on the specific choice made for the secondary grades. When the secondary grades equal to one, the resulting T2 fuzzy sets are called interval T2 fuzzy sets (IT2FS). In other words, the uniform shading over the entire FOU represents uniform weighting (possibilities) and such set is called an IT2FS.

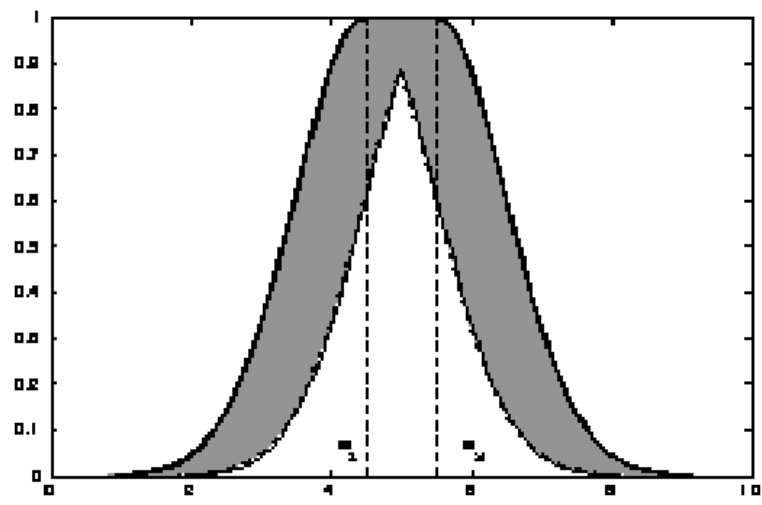

Fig. 5 FOU of Gaussian (primary) MF with Uncertain Mean
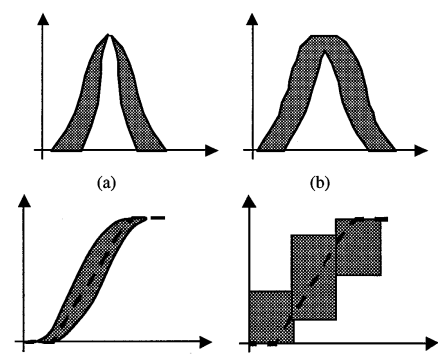

Fig. 6 FOU's: (a) Gaussian MF with Uncertain Standard Deviation (b) Gaussian MF with uncertain Mean (c) Sigmoidal MF with Inflection Uncertainties (d) Granulated Sigmoidal MF with Granulation Uncertainties 


\section{B. Interval Type-2 Fuzzy Sets}

An interval T2 fuzzy set (IT2FS) $\tilde{A}$ is characterized vide

(5), $\tilde{A}=\int_{x \in X} \int_{\left.u \in J_{x} \subseteq 0,1\right]} 1 /(x, u)=\int_{x \in X}\left[\int_{\left.u \in J_{x} \subseteq 0,1\right]} 1 / u\right] x$

where $x$, the primary variable in domain $X ; u \in U$, the secondary variable in domain $J_{x}$ at each $x \in X ; J_{x}$ is called the primary membership of $x$, defined in (5) while the secondary grades of $\tilde{A}: X \rightarrow\{[a, b]: 0 \leq a \leq b \leq 1\}$. Uncertainty about $\tilde{A}$ is conveyed by the union of primary memberships and termed as footprint of uncertainty (FOU) $\tilde{A}$, defined by (6),

$$
\operatorname{FOU}(\tilde{A})=\bigcup_{\forall x \in X} J_{x}=\left\{(x, u): u \in J_{x} \subseteq[0,1]\right\}
$$

The upper membership function (UMF) and lower membership function (LMF) of $\tilde{A}$ are two T1 MFs that bound the FOU (see figure 7). The UMF is associated with the upper bound of $F O U(\tilde{A})$, denoted by $\bar{\mu}_{\tilde{A}}(x)$ and described in (7) while the LMF is associated with the lower bound of $\operatorname{FOU}(\tilde{A})$, denoted by $\underline{\mu}_{\tilde{A}}(x)$, and described in (8).

$$
\bar{\mu}_{\tilde{A}}(x) \equiv \overline{F O U(\tilde{A})} \quad \forall x \in X
$$

$$
\underline{\mu}_{\tilde{A}}(x) \equiv \underline{F O U(\tilde{A})} \quad \forall x \in X
$$

Note that $J_{x}$ is an interval set, i.e.

$$
J_{x}=\left\{(x, u): u \in\left[\underline{\mu}_{\tilde{A}}(x), \bar{\mu}_{\tilde{A}}(x)\right]\right\}
$$

$\operatorname{FOU}(\tilde{A})$ is finally expressed in (10)

$$
\operatorname{FOU}(\tilde{A})=\bigcup_{\forall x \in X}\left[\underline{\mu}_{\tilde{A}}(x), \bar{\mu}_{\tilde{A}}(x)\right]
$$

\section{TYPE-2 FUZZY SET ENTROPY}

The process of obtaining necessary information to perform segmentation leads to the correct selection of the regions of interest of the color image. The proposed work presents an application of the theory of fuzzy sets to evaluate the regions of interest with fixed accuracy. The terms fuzziness index [24] and entropy [25] provide the measurement of fuzziness in a set and are used to define the degree of uncertainty [26] of the segmentation process. To measure the fuzziness of images, a few formal definitions are discussed below.

An ordinary fuzzy set $A$ of the universe of discourse $X$ is classically defined by its membership function

$\mu_{\mathrm{A}}(x): \mu_{A}: X \rightarrow[0,1], x \in X$

(11)
A point $x$ for which $\mu_{\mathrm{A}}(x)=0.5$ is said a crossover point of fuzzy set $A \subseteq X$.

The uncertainty is represented by the " $\alpha$-cut" of fuzzy set $A$ where a threshold $\alpha \in[0,1]$ is considered and the membership function can be defined as $\mu_{A}^{\alpha}(x)$ (classical set $A^{\alpha}$ or $\alpha$-cut of the fuzzy set $A$ ):

$\mu^{\alpha}{ }_{\mathrm{A}}(x): X \ddagger\{0,1\}$

$\mu^{\alpha}{ }_{A}(x)=1$ if $\forall x \geq \alpha=0$ if $\forall x<\alpha$ where $x \in X$

The fuzziness index $\gamma(\mathrm{A})$ of a fuzzy set $A$ reflects the degree of ambiguity by measuring the distance $d\left(A, A^{0.5}\right)$ between $A$ and its nearest set $A^{0.5}(\alpha=0.5)$ defined as:

$\gamma(A)=2 \times d\left(A, A^{0.5}\right) / n^{1 / p}$

(13)

A positive scalar $p$ is introduced to make $\gamma(A)$ lie between zero and one and its value depends on the type of distance function used. In the proposed algorithm with the help " $\alpha$-cut" we have used the " $n$-cut" of the fuzzy set. Here in (13), $n$ is the number of elements of $n$-cut vector. This measure represents the area between two membership functions $\mu_{A}(x)$ and $\mu_{A}^{\alpha}(x)$, described in (14).

$\gamma(A)=\lim _{\Omega \rightarrow X}\left(\frac{1}{\|\Omega\|} * \int_{\Omega}\left|\mu_{A}(x)-\mu_{A}^{0.5}(x)\right| d x\right)$

where $\|\Omega\|$ represents the size of the set $\Omega$ ( linear index values )In practice we can use the discrete formula as

$\gamma_{A}^{p}=\left[\frac{1}{\|X\|} * \sum_{x \in X}\left|\mu_{A}(x)-\mu_{A}^{0.5}(x)\right|^{p}\right]^{\frac{1}{p}}$

$\gamma_{A}^{p}$ is a monotonic function, where $p \in[1,+\propto]$ and $\|X\|$ represents the cardinality of the set $X$. When analytic ethods are used, $p=2$ (Euclidean distance) corresponds to the minimum value that makes $\left|\mu_{A}(x)-\mu_{A}^{0.5}\right|^{p}$ derivable. But when we consider the numeric methods the value of $p=1$ (Hamming Distance) is good.

The term entropy of fuzzy set $A$ which is denoted as $H(A)$ (monotonic increasing function) was first introduced by De Luca and Termini [27], expressed in (17).

$H(A)=\left(\sum S_{n}\left(\mu_{A}(x)\right)\right) / n \cdot \ln 2$

Where $S_{n}\left(\mu_{A}(x)\right)=-\mu_{A}(x) \ln \left(\mu_{A}(x)\right)-\left(1-\left(\mu_{A}(x)\right) \ln \left(1-\mu_{A}(x)\right)\right)$ In this work, we use the extension of the "De Luca and Termini" measure [28] to discrete images, proposed by Pal [53] for adapting to our problem. For an $M \times N$ image subset $A \subseteq X$ with $L$ gray levels $g \in\left[0, L^{-1}\right]$, the histogram $h(g)$ and the membership function $\mu_{X}(g)$ (consists of $\mu_{u}(g)$ and $\left.\mu_{l}(g)\right)$ the (linear) index of fuzziness is defined in (18) and shown in figure 10 .

$\gamma(A)=\frac{1}{M N} \sum_{g=0}^{L-1} h(g) *\left[\mu_{u}(g)-\mu_{l}(g)\right]$ 
Entropies in gray scale image segmentation [29] are already used with T2 fuzzy sets. It is nothing but the extension of works proposed by Tizhoosh [30].

Tizhoosh [31] applied T2 fuzzy sets for gray scale image thresholding and obtain good results with very noisy images. As proposed in [32], he used interval T2 fuzzy sets with the following "FOU":

Upper Limit: $\quad \mu_{u}(x): \mu_{u}(x)=[\mu(x)]^{0.5}$

Lower Limit: $\mu_{l}(x): \mu_{l}(x)=[\mu(x)]^{2}$

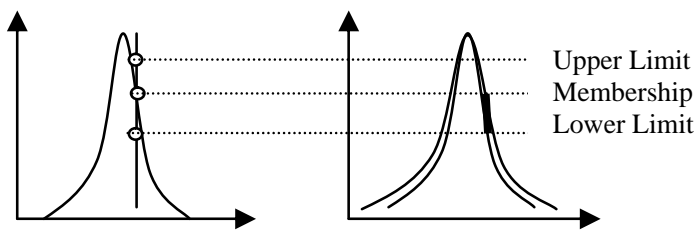

Fig. 7 Membership function for image processing

Here for the CMYK color model the same functions are used for image segmentation. To handle the drawback of gray scale imaging, in the proposed algorithm various correctional measures are considered. The proposed algorithm for the CMYK color image segmentation based on $\mathrm{T} 2$ fuzzy sets to measuring the fuzziness is presented below.

\section{A. Algorithm for Color Image Segmentation}

Begin

Step1: $\quad$ Read the JPEG file to be segmented

Step2: $\quad$ Select the proper shape of the interval base type-2 fuzzy set MF as

$$
A=\int_{\mathrm{x} \in \mathrm{X}} \int_{\mathrm{u} \in \mathrm{Jx}} \mu_{A}(x, u) /(x, u) J_{x} \subseteq[0,1]
$$

Step3: $\quad$ Fix the image size of $M \times N$ matrix;

Step4: Calculate (18) $h(g)$ for each color component of the color space; //For linear index calculation

Step5: Calculate " $n-$ cut" of the total image //for color pattern possibility matching with CMYK;

Step6: Initialize the position of the type-2 MF;

Step7: Shift the MF with gray level ranges;

Step8: Mapping the picture colors into gray scale format; IIFor contour detection

Step 9: $\quad$ Calculate the values of MF : $\mu_{u}(g)$ and $\mu_{l}(g)$

(where $\mu_{u}(g), \mu_{l}(g) \subseteq \mu_{X}(\mathrm{~g})$ ) in each position;

Step10: Compute the edge detection of the image based on contour formation;

Step11: Compute similarity matrix, say ' $W$ ' based on inverting contours;

Step12: Find mid, max and min of fuzzy index;

Step13: Compute " $n$-cut" eigenvectors; Ilpossible combination of colors for the input picture

Step14: Threshold the image with thresholding value $\gamma_{\max }$;

Ilunwanted pixel elimination based on Median filter

Step15: Masking of segmented image using $m s k$ matrix is defined by

$$
\text { [ } \quad 0000000
$$

$$
m s k=
$$

01110

000000

Step16: Median filtering on the segmented image to remove noise; Step17: Apply the region merging process using the acquired classes of pixels; segmented portion of images are merged Step18: Smoothing of image to reduce the number of connected components;

Step19: Calculate the connected components; " $n-c u t$ " Eigenvector Step20: Calculate the number of pixels in the final image and store in a variable and compare with the original one;

End.

\section{EXPERIMENTAL RESULTS}

In order to test the performance of the algorithm, the sample (figure 11, 12) are taken in JPEG image format having a size of $132 \times 130$ pixels in RGB color mode. The results of execution of the algorithm is as follows:-

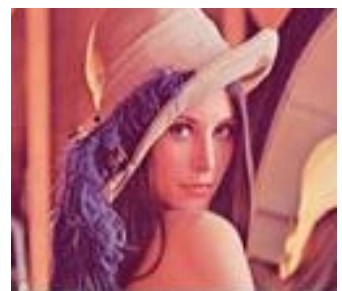

Fig-8 Original image

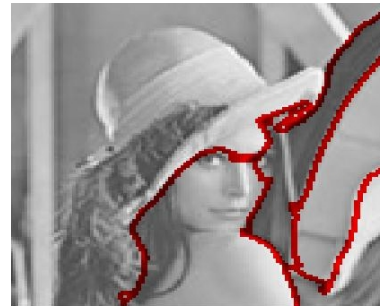

Fig -10 Contour Detection

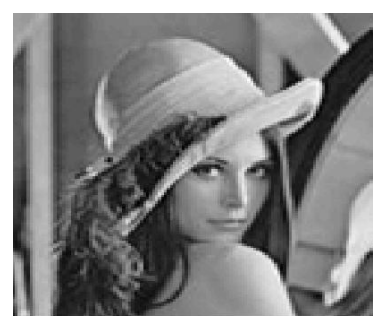

Fig -12 Image in grey scale

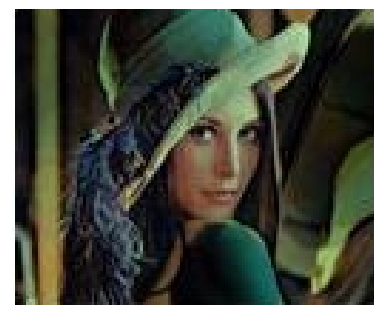

Fig -13 Hue saturated image

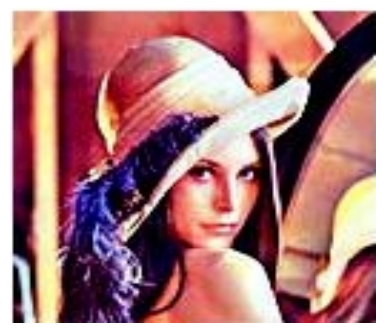

Fig -15 Intensed sharp image

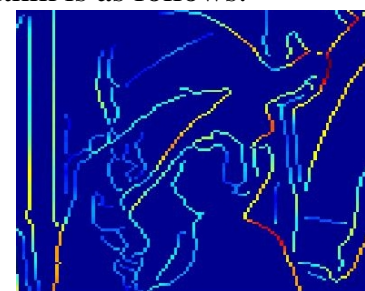

Fig -9 Segmented Image

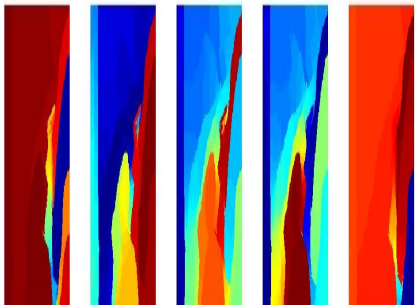

Fig -11

$\mathrm{N}$-cut eigenvectors

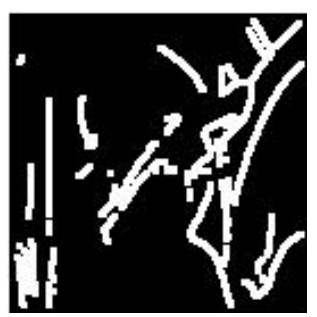

Fig -13 Grey scale segmentation

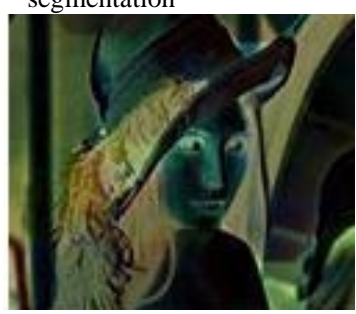

Fig -14 Inverse hue intensed image

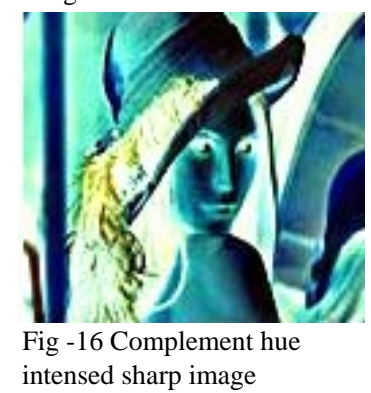




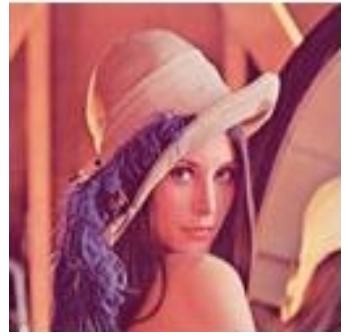

Figure -17 Enhanced image in CMYK model

In the above figures it clearly shows how the picture can be enhanced in CMYK model.

\section{A. Type-2 fuzzy sets Entropy vs. type-1 fuzzy sets Entropy}

The histogram of the color component of an image can be separated into number of peaks each corresponds to a region, and there exits a threshold at the valley between any two adjacent peaks. Fuzzy entropy represents the fuzziness of the color component of the images based on information theory and fuzzy logic and used as a criterion to select the thresholds automatically. While shifting the membership function of a fuzzy set on the histogram of a color component of the image, the fuzzy entropy values of the histogram are obtained considering the neighboring information among pixels. We employ both the local and global information in this regard. Time complexity of the proposed algorithm has been computed and compared with T1 fuzzy sets entropy approach.

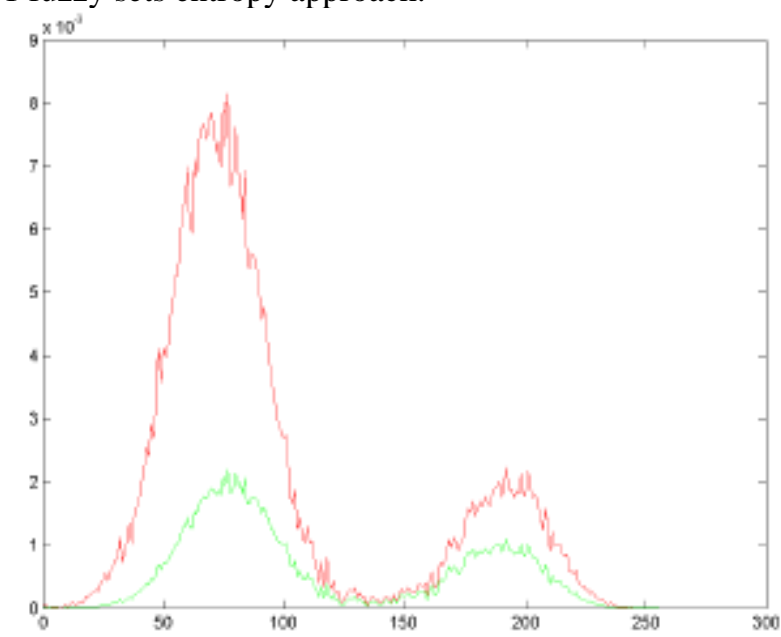

Fig -18 Type -2 Fuzzy set entropy Vs Type - 1 fuzzy set entropy

In figure 18, the red graph shows T2 entropy while the green graph shows T1 entropy class. Therefore, the results of segmentation for $\mathrm{T} 2$ is more appropriate than $\mathrm{T} 1$.

\section{CONCLUSION}

Color image segmentation is a difficult task in image processing. Any unique algorithm is certainly never established to tackle all kinds of images. We have tried to apply a new algorithm provided by fuzzy set theory and approximations. The idea was to introduce the application of type-2 fuzzy sets, to take into account the total amount of uncertainty present at the segmentation stage such as fuzziness, discord and non-specificity. The examples have demonstrated the applicability of this algorithm. In the proposed algorithm the time complexity of segmentation is $\mathrm{O}(\mathrm{n})$ and therefore linear while that of in normal mode is $\mathrm{O}(\mathrm{n} 2)$. The number of replicate pixels is calculated. In normal segmentation the number of pixels are reduced but in here it has been enhanced.

\section{ACKNOWLEDGMENT}

I would like to thank Prof.Dr.Jaya Sil Head of the Department of IT of Bengal Engineering and Science Univeristy, Howrah, India for spending her valuable time and giving me such a wonderful guidance to create my interest in this line.

\section{REFERENCES}

[1] J. Mendel, Uncertain Rule-Based Fuzzy Logic Systems: Introduction and New Directions. Upper Saddle River, NJ: Prentice-Hall, 2001.

[2] J. Mendel and R. John, "Type-2 fuzzy set made simple," IEEE Trans.Fuzzy Syst., vol. 10, no. 2, pp. 117-127, Apr. 2002

[3] J. Mendel, "Computing derivatives in interval type-2 fuzzy logic systems,'IEEE Trans. Fuzzy Syst., vol. 12, no. 1, pp. 84-98, Feb. 2004.

[4] J. Mendel and R. John, "A fundamental decomposition of type-2 fuzzy sets," in Proc. 2001 Joint Conf. IFSA/NAFIPS, Jul. 2001, pp.1896-1901

[5] Q. Liang and J. Mendel, "MPEG VBR video traffic modeling and classification using fuzzy techniques," IEEE Trans. Fuzzy Syst., vol. 9, no.1, pp. 183-193, Feb. 2001

[6] "Overcoming time-varying co-channel interference using type-2 fuzzy adaptive filter," IEEE Trans. Circuits Syst. II, Analog Digital Signal Process., vol. 47, pp. 1419-1428, Dec. 2000

[7] Q. Liang, N. Karnik, and J. Mendel, "Connection admission control in ATM networks using survey-based type-2 fuzzy logic systems," IEEE Trans. Syst., Man, Cybern. C, Appl. Rev., vol. 30, pp. 329-339, Aug. 2000.

[8] H. Hagras, "A hierarchical type-2 fuzzy logic control architecture for autonomous mobile robots," IEEE Trans. Fuzzy Syst., vol. 12, no. 4, pp. 524-539, Aug. 2004

[9] K.Wu, "Fuzzy interval control of mobile robots," Comput. Elect. Eng., vol. 22, pp. 211-229, 1996.

[10] R. Yager, "Fuzzy subsets of type II in decisions," J. Cybern., vol. 10, pp. 137-159, 1980.

[11] J. Chaneau, M. Gunaratne, and A. Altschaeffl,, J. Bezdek, Ed., "An application of type-2 fuzzy sets to decision making in engineering," in Analysis of Fuzzy Information, vol. II: Artificial Intelligence and DecisionSystems. Boca Raton, FL: CRC, 1987.

[12] Q. Liang and J. Mendel, "Equalization of nonlinear time-varying channels using type-2 fuzzy adaptive filters," IEEE Trans. Fuzzy Syst., vol. 8, no. 5, pp. 551-563, Oct. 2000.

[13] I. Turksen, "Interval-representation of sets: DempsterPawlak-Turksen schema," in Proc. IEEE Int. Conf. Fuzz Syst., May 2002, vol. 1, pp. 602-606.

[14] N. Karnik and J. Mendel, "Applications of type-2 fuzzy logic systems to forecasting of time series," Inform. Sci., vol. 120, pp. 89-111, 1999.

[15] P. Innocent, R. John, I. Belton, and D. Finlay, "Type 2 fuzzy representations of lung scans to predict pulmonary emboli," in Proc. 2001 IFSA/NAFIPS Joint Conf., Vancouver, BC, Canada, Jul. 2001, pp. 1902-1907.

[16] R. John, "Type 2 fuzzy sets for knowledge representation and inferencing," in Proc. IEEE Int. Conf. Fuzzy Syst., May 1998, vol. 2, pp. 1003-1008.

[17] R. John and C. Czarnecki, "A type 2 adaptive fuzzy inferencing system," in Proc. Int. Conf. Syst., Man, Cybern., Oct. 1998, vol. 2, pp. 2068-2073.

[18] O. Uncu and I. Turksen, "A new two-step fuzzy inference approach based on Takagi-Sugeno inference using discrete type 2 fuzzy sets," in Proc. 2003 Int. Conf. NAFIPS, July 2003, pp. 32-37.

[19] R. John and C. Czarnecki, "An adaptive type-2 fuzzy system for learning linguistic membership grades," in Proc. Int. Conf. Fuzzy Syst., Aug. 1999, vol. 3, pp. 1552-1556.

[20] S. Auephanwiriyakul, A. Adrian, and J. Keller, "Type 2 fuzzy set analysis in management surveys," in Proc. Int. Conf. Fuzzy Syst.,May 2002, vol. 1, pp. 1321-1325.

[21] R. John, P. Innocent, and M. Barnes, "Type 2 fuzzy sets and neurofuzzy clustering of radiographic tibia images," in Proc. Int. Conf. FuzzySyst., May 1998, vol. 2, pp. 1373-1376.

[22] P. Innocent and R. John, "Type 2 fuzzy diagnosis," in IEEE Int. Conf.Fuzzy Syst., May 2002, vol. 2, pp. 1326-1330. 
[23] R. John, "Type-2 inferencing and community transport scheduling," in Proc. 4th Euro. Congr. Intelligent Techniques Soft Computing, Aachen, Germany, Sep. 1996, pp. 1369-1372.

[24] I. Ozkan and I. Turksen, "Entropy assessment for type-2 fuzziness," in Proc. Int. Conf. Fuzzy Syst., Jul. 2004, vol. 2, pp. 1111-1115.

[25] F. Rhee and C.Hwang, "Atype-2 fuzzy C-means clustering algorithm," in Proc. Joint Conf. IFSA/NAFIPS, Jul. 2001, pp. 1926-1919.

[26] "An interval type-2 fuzzy perceptron," in Proc. Int. Conf. Fuzzy Syst., May 2002, vol. 2, pp. 1331-1335.

[27] "An interval type-2 fuzzy K-nearest neighbor," in Proc. Int. Conf. Fuzzy Syst., May 2003, vol. 2, pp. 802-807.

[28] C. Hwang and F. Rhee, "An interval type-2 fuzzy C spherical shells algorithm," in Proc. Int. Conf. Fuzzy Syst., Jul. 2004, vol. 2, pp. 1117-1122.

[29] R. Krishnapuram, O. Nasraoui, and H. Frigui, "The fuzzy C-spherical shells algorithm," IEEE Trans. Neural Netw., vol. 3, no. 5, pp. 663-671,

[30] Sep. 1992

[31] J. Tou and R. Gonzalez, Pattern Recognition Principles. Reading, MA: Addison-Wesley, 1974

[32] J. Bezdek, Pattern Recognition with Fuzzy Objective Function Algorithms. New York: Plenum, 1981.

[33] A. Bigand, T. Bouwmans, J.P. Dubus "Extraction of line segments from fuzzy images," Pattern Recognition Letters, vol. 22, pp. 1405-1418, 2001

[34] R. Demirci, "Rule-based automatic segmentation of color images," Int J. Electron. Commun., vol. 60, pp. 435-442, 2006.

Saikat Maity (M'77) received the B.TECH. from Institute of Engineering and Technology under Kalyani University(2000) and M.TECH. degree from Calkcutta University(2004), Calcutta, West Bengal, India in computer science and engineering, respectively.

$\mathrm{He}$ is an Associate Professor at the Department of Computer Science and Engineering at Dr.B.C.Roy Engineering College under West Bengal University and Technology, Calcutta, India.

He has research experience in the soft computing,image processing,, fuzzy logic controllers, and other intelligent systems. He is currently working on research supported by Bengal Engineering and College University

Dr.Jaya Sil received the B.E in Electronics and Telecommunication (84), M.E. degree in Computer Science \& Engineering (86), Ph.D in Computer Science (96) respectively.

Dr.Jaya Sil is with Bengal Engineering Science \& University, Howrah, India.She is now Head of the department in the Department of Computer Science and Engineering in B.E.S.U and also the member of IEEE. Her area of research are Soft Computing Techniques, Image Processing, Bio-Informatics and Multi Agent Systems.She is also the Chairperson, Network Implementing Committee, TEQIP, Member Secretary, Ph.D. Committee, Director, School of VLSI Technology, Member of Space Committee, Member of enquiry Committee, Member of the committee to look after the proposed introduction of Aerospace Engineering. She is now in association with Heidelberg university, Germany, Jadavpur University. 